\title{
Analysis of clinicopathological features and prognostic factors of non-Hodgkin's intravascular large B-cell lymphoma
}

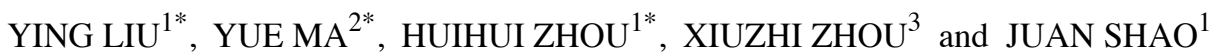 \\ ${ }^{1}$ Department of Oncology, Pathology and Dermatology, Yantai Yuhuangding Hospital of Qingdao University, Yantai, \\ Shandong 264000; ${ }^{2}$ Department of Urology, Mianyang Central Hospital, Mianyang, Sichuan $621000 ;{ }^{3}$ School of \\ Basic Medical Sciences, Binzhou Medical University, Yantai, Shandong 264003, P.R. China
}

Received August 28, 2019; Accepted May 7, 2020

DOI: $10.3892 / 01.2020 .11908$

\begin{abstract}
Non-Hodgkin's intravascular large B-cell lymphoma is a highly invasive extranodal lymphoma. The proliferating tumor cells invade the small vessels and capillaries of different organs. The clinical symptoms are atypical, there is lack of specificity, and the molecular and biological behaviors are not clear, thus, the present study aimed to improve the current understanding of non-Hodgkin's intravascular large B-cell lymphoma (IVL) and provide an accurate basis for clinical treatment and prognosis, by retrospectively analyzing and summarizing the clinicopathological features, immunohistochemical findings and molecular characteristics of 17 patients with IVL. The Kaplan-Meier method and log rank test were implemented to determine survival outcomes. Fisher's exact test was used to determine the association between clinicopathological features and the expression levels of Ki-67, c-Myc, B-cell lymphoma 6 (Bcl-6) and B-cell lymphoma 2 (Bcl-2), while multivariate Cox regression analysis was performed to identify the independent risk factors that affect the survival rates of patients with IVL. $\mathrm{P}<0.05$ was considered to indicate a statistically significant difference. Among the 17 patients with IVL, 13 cases (76.47\%) occurred in the adrenal gland and four cases $(23.53 \%)$ occurred on the skin demonstrated positive IgH gene rearrangement. FISH analysis indicated that cleavage of the c-Myc gene was closely associated with sex, hypertension status and tumor size, while cleavage of the
\end{abstract}

Correspondence to: Professor Xiuzhi Zhou, School of Basic Medical Sciences, Binzhou Medical University, 346 Guanhai Road, Yantai, Shandong 264003, P.R. China

E-mail: zxz7810@163.com

Dr Juan Shao, Department of Oncology, Pathology and Dermatology, Yantai Yuhuangding Hospital of Qingdao University, 20 Yuhuangding East Road, Yantai, Shandong 264000, P.R. China E-mail: 187205259@qq.com

*Contributed equally

Key words: non-Hodgkin's intravascular large B-cell lymphoma, clinicopathological features, prognosis, molecular characteristics
Bcl-6 gene was closely associated with tumor size parameters. Overall, the results suggest that the Ki-67 proliferation index is an independent risk factor for the prognosis (survival time) of patients with IVL.

\section{Introduction}

Non-Hodgkin's intravascular large B-cell lymphoma (IVL) is an extremely rare subgroup of diffuse large B-cell lymphoma, accounting for only $1 \%$ of the total cases of the disease (1). According to the latest histological classification of lymphomas, the World Health Organization (WHO) classified IVL as an extranodal lymphoma in 2001 (1-3). IVIBCL, together with primary effusion lymphoma and large B cell lymphoma of the mediastinum, was classified as a subtype of DLBCL $(2,3)$. According to the new classification standard (2), IVLBCL belongs to extranodal lymphoma, which is characterized by diffuse and obliterative proliferation of lymphoma cells in tissues, organs and lumens of small and medium vessels (terminal arteries, veins, capillaries and blood sinusoid), and involvement of different extranodal organs and tissues, including the central nervous system, skin, lung, kidney, adrenal gland and bone marrow (2-5). It does not involve lymph nodes and peripheral blood, and has several clinical manifestations, including fever, large liver and spleen, hemocytopenia, disseminated intravascular coagulation and organ damage (2-5). The reported incidence rate of men and women worldwide is $\sim 1: 1$, the median age of onset is $\sim 60$ years and the average survival time is $\sim 6-9$ months (1-5). Most patients die within 1 year, and it is a malignant tumor with poor prognosis (1-5). IVL is a highly invasive and extremely rare malignant hematological disease, with poor prognosis and a lack of specificity in the clinical setting $(1,2,4)$. The stable immunohistochemical expression of CD34, CD20 and PAX5 is considered beneficial to the diagnosis and differential diagnosis of IVL $(2,3)$, which may occur in various organs and tissues, though are most commonly found in the adrenal gland and skin tissue (3). As the nomenclature and biological characteristics of IVL remained unclear for a long period of time, diagnosis and treatment strategies for IVL were not standardized in the clinical setting, including imaging and pathological methodologies (3). The principle clinical manifestations included: Fever in 13 cases $(76.47 \%)$, nephrotic syndrome in four 
cases $(23.53 \%)$, hypertension in six cases $(35.29 \%)$, elevated serum lactic dehydrogenase in nine cases $(52.94 \%)$, increased interleukin 6 expression in seven cases $(41.18 \%)$, tumor size $\leq 2 \mathrm{~cm}$ in five cases $(29.41 \%)$ and tumor size $>2 \mathrm{~cm}$ in 12 cases (70.59\%). Immunohistochemistry analysis demonstrated that the positive expression rates of $\mathrm{CD} 34, \mathrm{CD} 20$, paired box protein PAX5, CD10, Mum-1, Bcl-2, Bcl-6 and c-Myc in the 17 patients were $17 / 17,17 / 17,17 / 17,8 / 17,9 / 17,7 / 17,8 / 17$ and $9 / 17$, respectively. The positive expression rates of $\mathrm{Ki}-67$ were $>60 \%$ in all patients, and $>70 \%$ in 11 patients. No expression was observed for CD3, CD6, granzyme B, EBER, CD113, CD33, CD117, CKpan and HMB-45. Fluorescence in situ hybridization (FISH) analysis demonstrated that of the 17 patients with IVL, seven cases $(41.18 \%)$ exhibited c-Myc cleavage, eight cases (47.06\%) revealed Bcl-2 cleavage, seven cases (41.18\%) exhibited Bcl-6 cleavage and 16 cases $(94.12 \%)$ Thus, the present study aimed to improve the current understanding of IVL and provide an accurate basis for clinical treatment and prognosis, via $\mathrm{HE}$ morphology, immunohistochemistry, FISH detection and gene rearrangement, by retrospectively analyzing and summarizing the clinicopathological and pathologic features, and follow-up data of 17 patients with IVL.

\section{Materials and methods}

Patient data. A total of 17 IVL samples (13 men and 4 women; age range, 38-82 years; median age, 59 years; mean age, 57.2 years) were collected following surgical resection at the Yantai Yuhuangding Hospital (six cases), Shandong Provincial Hospital (five cases) and the Affiliated Hospital of Qingdao University (six cases) between January 2000 and December 2018. Diagnoses were pathologically confirmed by three senior pathologists from the Department of Pathology, Yantai Yuhuangding Hospital of Qingdao University (Yantai, China), using a BX53 multi-head light microscope (Olympus Corporation), set at magnifications of $\times 4, x 100$ and $\times 200$. The clinical data and general findings were acquired from clinical medical records and specimen delivery forms. The follow-up information was obtained by telephone, from medical record rooms in the aforementioned hospitals and from the household registration department of the Public Security Bureau (Yantai, Qingdao and Jinan; China).

Immunohistochemistry (IHC). IVL tissue samples were fixed in $4 \%$ neutral formaldehyde for 6-48 $\mathrm{h}$ at room temperature, embedded in paraffin and cut into $4-\mu \mathrm{m}$-thick sections, prior to staining with hematoxylin for $90 \mathrm{sec}$ at room temperature and eosin for $3 \mathrm{sec}$ at room temperature. For each case, representative wax blocks were selected for histochemical staining, using the detailed steps of immunohistochemistry, as follows: The sections were deparaffinized with xylene at room temperature for $10 \mathrm{~min}$ and washed twice with buffer for $3 \mathrm{~min}$. The sections were incubated with hydrogen peroxide at room temperature for $10-15 \mathrm{~min}$ to inhibit endogenous peroxidase activity, washed twice with buffer for $5 \mathrm{~min}$ and subsequently incubated with ultra V block (Guangzhou Anbiping Pharmaceutical Technology Co., Ltd.) at room temperature for $5 \mathrm{~min}$. Tissue sections were re-washed twice with buffer for $5 \mathrm{~min}$, prior to incubation with primary antibody dilution (Guangzhou Anbiping Pharmaceutical
Technology Co., Ltd.) at $37^{\circ} \mathrm{C}$ for $1-2 \mathrm{~h}$, and subsequently washed twice with buffer for $5 \mathrm{~min}$. Subsequently, tissue sections were incubated with primary antibody enhancer (Guangzhou Anbiping Pharmaceutical Technology Co., Ltd.) at room temperature for $20 \mathrm{~min}$, and washed twice with buffer for $5 \mathrm{~min}$. This was followed by incubation with enzyme labeled secondary antibody (Guangzhou Anbiping Pharmaceutical Technology Co., Ltd.) at room temperature for $30 \mathrm{~min}$, and sections were subsequently washed twice with buffer for $5 \mathrm{~min}$. DAB Plus Chromogen (1-2 drops) was added to $1 \mathrm{ml}$ DAB Plus Substrate (both purchased from Guangzhou Anbiping Pharmaceutical Technology Co., Ltd.). The mixture was added to the slides and incubated at $37^{\circ} \mathrm{C}$ for $3-15 \mathrm{~min}$. The slides were flushed with distilled water for $3 \mathrm{~min}$, and counterstained with hematoxylin (10:100) for $4 \mathrm{~min}$ and lithium carbonate aqueous solution $(5: 100)$ for $10 \mathrm{~min}$ at room temperature. Subsequently, the sections were dehydrated with $85 \%$ alcohol, $95 \%$ alcohol and $100 \%$ alcohol for 2 min each at room temperature. The slides were flushed with xylene at room temperature for $2 \mathrm{~min}$ and subsequently mounted onto coverslips, using mounting medium (all purchased from Guangzhou Anbiping Pharmaceutical Technology Co., Ltd.) Cells were observed under the BX53 multi-head light microscope (Olympus Corporation; magnification, x100). Antibodies against CD34 (1:200; cat. no. MAB-0532), CD20 (1:200; cat. no. MAB-0594), PAX-5 (1:400; cat. no. MAB-0332), CD10 (1:200; cat. no. MAB-0542), Interferon regulatory factor 4 (IRF4; 1:200; cat. no. MAB-2232), B-cell lymphoma 2 (Bcl-2; 1:400; cat. no. MAB-2532), B-cell lymphoma 6 (Bcl-6; 1:200; cat. no. MAB-0323), c-Myc (1:200; cat. no. MAB-7932), Ki-67 (1:200; cat. no. MAB-1132), CD3 (1:200; cat. no. MAB-0212), CD56 (1:400; cat. no. MAB-0582), granzyme B (GrB; 1:600; cat. no. MAB-6332), EBER (1:200; cat. no. MAB-8232), CD13 (1:200; cat. no. MAB-2132), CD33 (1:200; cat. no. MAB-2132), CD117 (1:200; cat. no. MAB-9932), CKpan (1:200; cat. no. MAB-8742) and HMB-45 (1:400; cat. no. MAB-5332), and kits (cat. no. MAB-00153) were purchased from Guangzhou Anbiping Pharmaceutical Technology Co., Ltd., using known positive sections as the positive control. The experimental steps were performed according to the protocol for each kit. CD34, CD20, CD10, CD3, cdl3, CD33, CD56 and Bcl-2 were all membrane-positive, while Pax-5, mum-1, c-Myc, Ki-67, EBER, cdll7 and bcl-6 were expressed in the nucleus, and GrB, CKpan and HMB-45 in the cytoplasm.

Fluorescence in situ hybridization (FISH). Tissue samples were cut into 3-4 um-thick sections and heated at $60^{\circ} \mathrm{C}$ for $2 \mathrm{~h}$. Sections were deparaffinized with xylene at room temperature for 10 min and incubated with $3 \% \mathrm{H}_{2} \mathrm{O}_{2}$ methanol solution in a humidified box at room temperature for $15 \mathrm{~min}$ to inhibit endogenous peroxidase activity, and subsequently washed three times with distilled water, for $3 \mathrm{~min}$ each time. Tissue sections were incubated with proteinase K (Guangzhou Anbiping Pharmaceutical Technology Co., Ltd.) in a humidified box at room temperature for $15 \mathrm{~min}$, and subsequently washed three times with PBS ( $\mathrm{pH}$ 7.4) specialized for in situ hybridization, for $5 \mathrm{~min}$ each time. Sections were then washed twice with distilled water, for 3 min each time. The pre-hybridizing solution (40:100, Guangzhou Anbiping Pharmaceutical Technology Co., Ltd.) was added and the box was incubated 


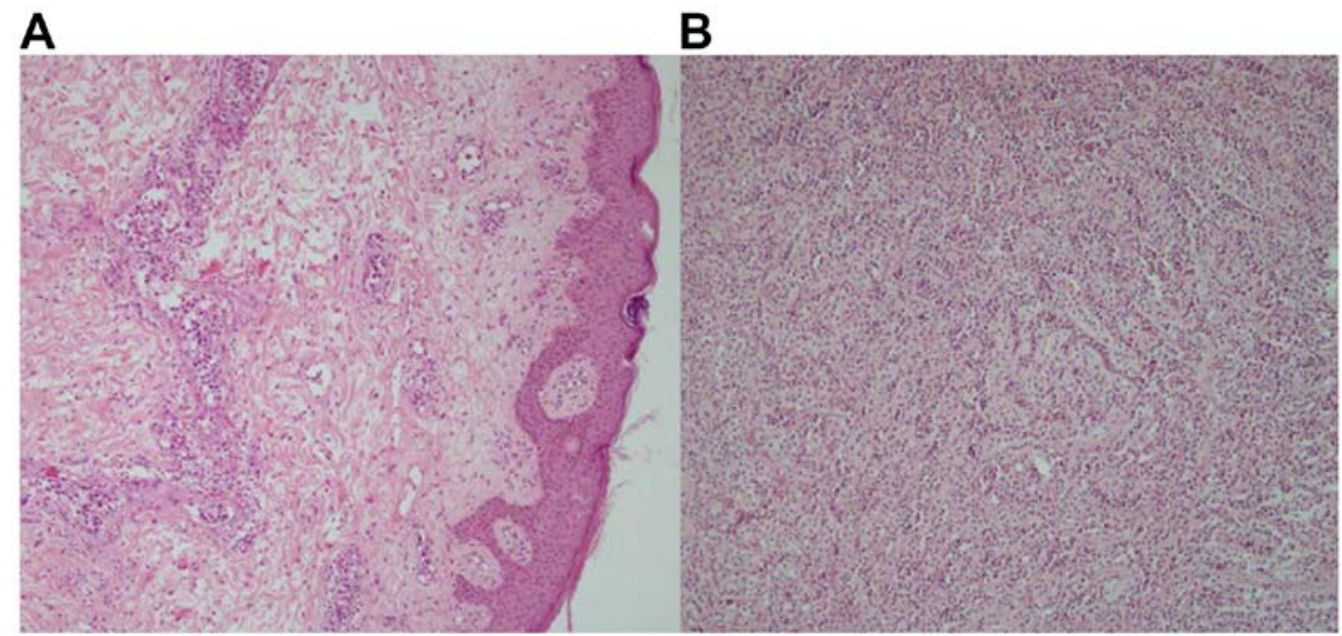

Figure 1. Histopathological analysis of IVL by hematoxylin \& eosin staining. Representative images of (A) adrenal IVL and (B) skin IVL (magnification, x100). IVL, Hodgkin's intravascular large B-cell lymphoma.

at $38^{\circ} \mathrm{C}$ for $3 \mathrm{~h}$. Subsequently, hybridizing solution (40:100, Guangzhou Anbiping Pharmaceutical Technology Co., Ltd.) was added and the wet box was incubated at $38^{\circ} \mathrm{C}$ for $3 \mathrm{~h}$ to block the non-specific background staining. Following removal of excess liquid, the hybridizing liquid (40:100, Guangzhou Anbiping Pharmaceutical Technology Co., Ltd.) was added, the tissues were overlaid with special FISH coverslips (Guangzhou Anbiping Pharmaceutical Technology Co., Ltd.), and the humidified box was incubated at $38^{\circ} \mathrm{C}$ overnight, then washed twice with 2X SSC buffer (Guangzhou Anbiping Pharmaceutical Technology Co., Ltd.) at $37^{\circ} \mathrm{C}$, for 5 min each time. Sections were subsequently washed with $0.5 \mathrm{X}$ SSC buffer for $15 \mathrm{~min}$ at $37^{\circ} \mathrm{C}$, and then re-washed twice with $0.2 \mathrm{X}$ SCC buffer, for 5 min each time, prior to adding blocking buffer (Guangzhou Anbiping Pharmaceutical Technology Co., Ltd.) at $-20^{\circ} \mathrm{C}$ for $30 \mathrm{~min}$.

c-Myc, Bcl-2 and Bcl-6 fracture probes and kits (cat. no. MCB-00153) from Guangzhou Anbiping Pharmaceutical Technology Co., Ltd., were used to confirm the cleavage of c-Myc, Bcl-2 and Bcl-6. Multicopy patient samples were used as positive controls, while normal lymph nodes were used as negative controls. The experiment was performed according to the manufacturers' protocols. In the case where separation of red and green signals was $>2$ signal points, it was considered a cleavage. A total of 200 tumor nuclear signals were recorded in the high-power visual field, and the ratio of isolated signaling cells to counting cells was $\geq 30 \%$, suggesting that the gene was broken. If the ratio of isolated signaling cells to counting cells was $<10 \%$, it suggested that the gene was not broken. When the ratio of isolated signaling cells to counting cells was $\geq 10 \%$ and $<30 \%$, a further 200 counting cells were added. The ratio of isolated signaling cells to counting cells was then recalculated and was confirmed to be positive if $\geq 15 \%$, and negative if $<15 \%$.

Gene rearrangement detection. DNA was extracted from the tissue samples using nucleic acid extraction buffer (Amoy Diagnostics Co., Ltd.) to detect gene rearrangement of the immunoglobulin heavy chain $(\operatorname{IgH})$. The detection fragments were selected according to the van Krieken method (4) and
PCR capillary electrophoresis was performed according to the instructions of the Gene Rearrangement Detection kit (Shanghai Yuanqi Co., Ltd.). The following thermocycling conditions were used for PCR: Initial denaturation at $95^{\circ} \mathrm{C}$ for $7 \mathrm{~min}$, low temperature annealing at $60^{\circ} \mathrm{C}$ for $45 \mathrm{sec}$ and $72^{\circ} \mathrm{C}$ for $90 \mathrm{sec}$ for a total of 40 cycles, and elongation at $72^{\circ} \mathrm{C}$ for $10 \mathrm{~min}$. The total reaction system was $20 \mu \mathrm{l}$ and the denatured products were analyzed via capillary electrophoresis on the gene analyze $r$ (room temperature extension). Results were interpreted according to the instructions on the kit purchased from Amoy Diagnostics Co., Ltd.

Statistical analysis. An Excel database of patients was established, and statistical analysis was performed using SPSS software (version 17.0; SPSS, Inc.). The association between clinicopathological features and the expression levels of Ki-67, c-Myc, Bcl-6 and Bcl-2 was evaluated using Fisher's exact test. Survival curves were generated and the survival rate was calculated using the Kaplan-Meier method. Multivariate Cox regression analysis was performed and the log-rank test was used to determine the independent risk factors affecting the survival of patients. $\mathrm{P}<0.05$ was considered to indicate a statistically significant difference.

\section{Results}

Clinicopathological data. Of the 17 patients with IVL, 13 cases (76.47\%) occurred in the adrenal gland (Fig. 1A), while four cases $(23.53 \%)$ occurred on the skin (Fig. 1B). The principle clinical manifestations included: Fever in 13 cases (76.47\%), nephrotic syndrome in four cases $(23.53 \%)$ and hypertension in six cases (35.29\%).

IHC analysis. The positive expression rates of CD34 (Fig. 2A), CD20 (Fig. 2B), paired box protein PAX5 (Fig. 2C), CD10, IRF4, Bcl-2, Bcl-6 and c-Myc in the 17 patients with IVL were $17 / 17,17 / 17,17 / 17,8 / 17,9 / 17,7 / 17,8 / 17$ and $9 / 17$, respectively. The positive expression rates of Ki-67 were $>60 \%$. Conversely, the expression rates of CD3, CD56, GrB, EBER, CD13, CD33, CD117, CKpan and HMB-45 were negative (data not shown). 


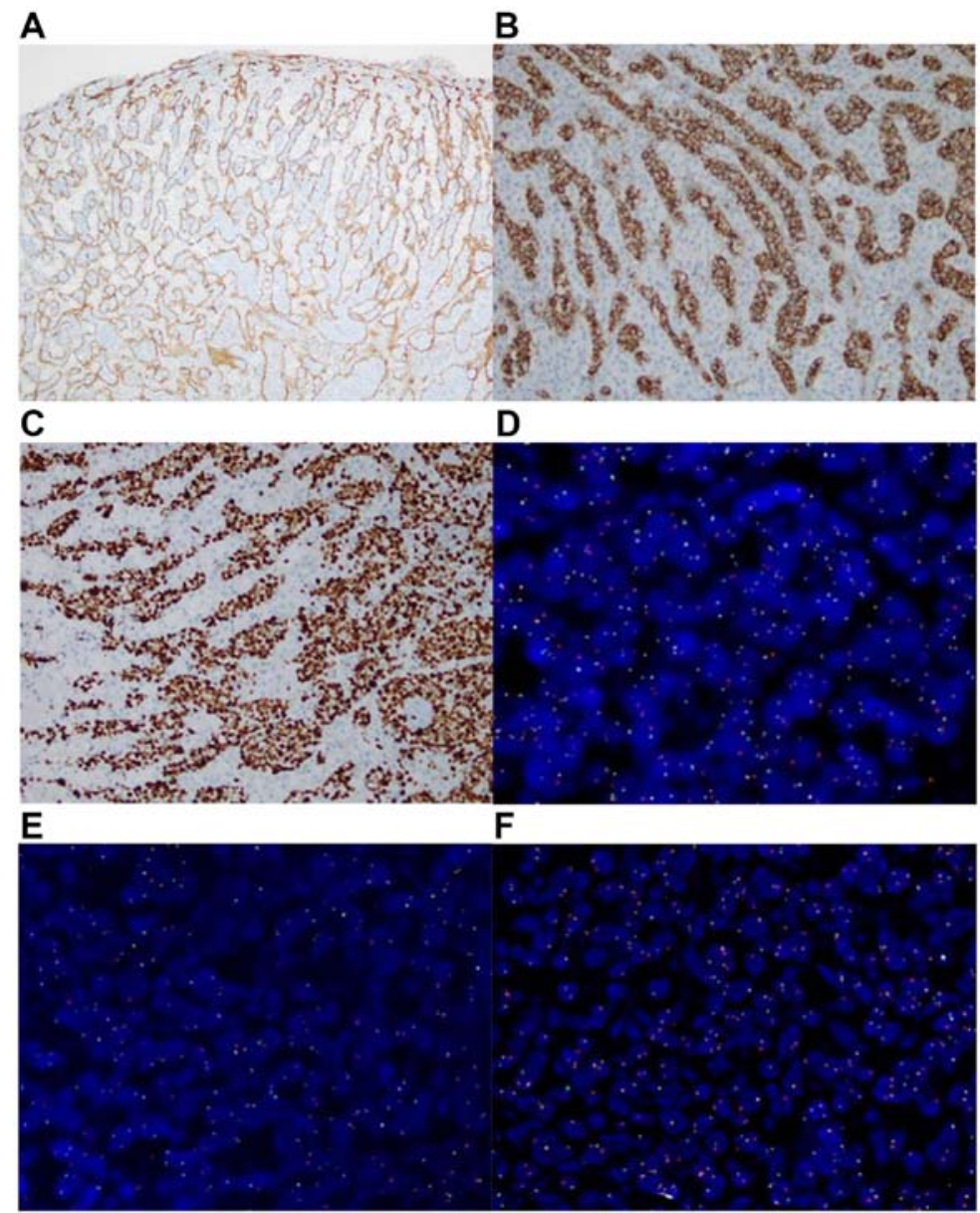

Figure 2. Protein detection via immunohistochemistry. Representative results of (A) CD34, (B) CD20 and (C) paired box protein PAX5 (all magnification, x200). Detection of gene cleavage by FISH. Representative results of (D) c-Myc, (E) Bcl-2 and (F) Bcl-6 (all magnification, x100). Bcl, B-cell lymphoma. FISH, Fluorescence in situ hybridization.

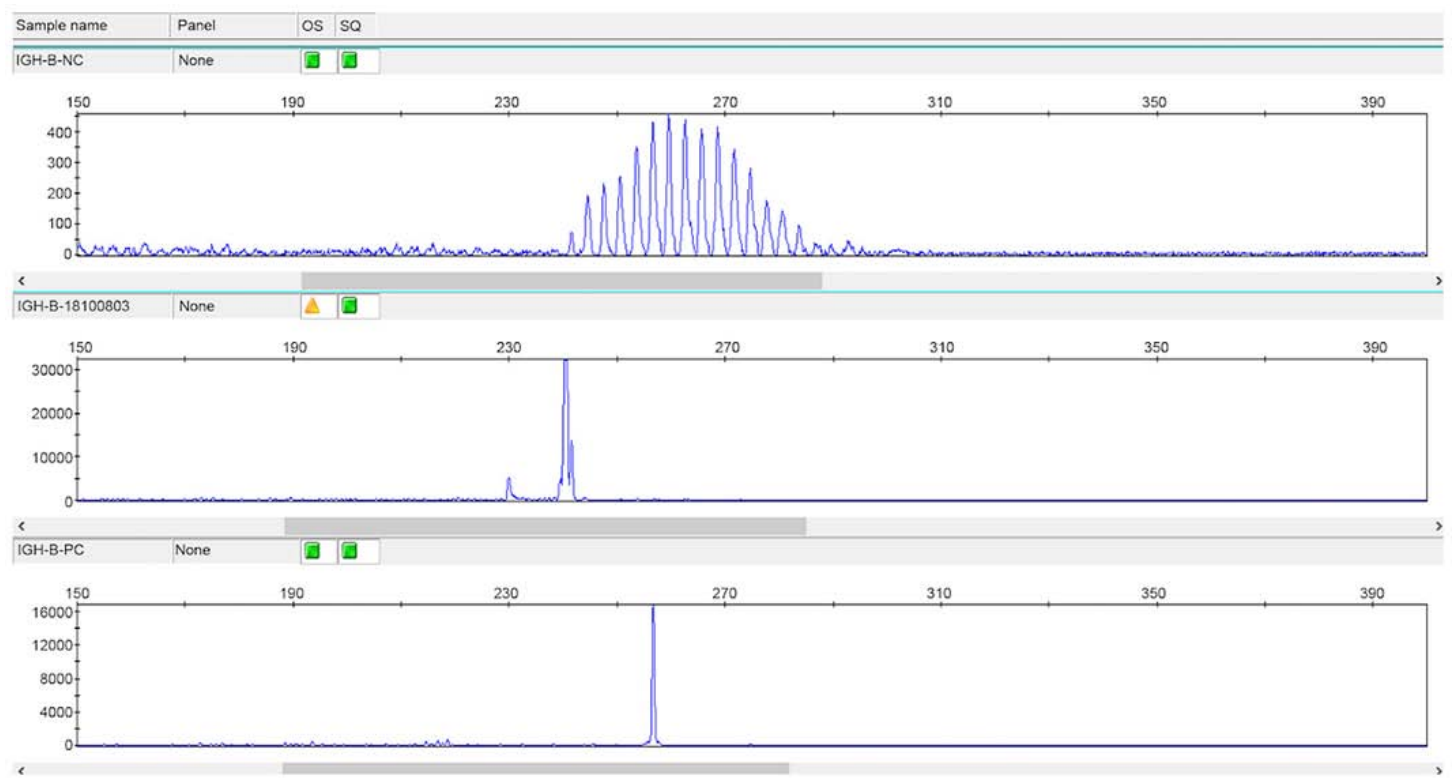

Figure 3. Detection of immunoglobulin gene rearrangement by PCR capillary electrophoresis.

Molecular characteristics. FISH analysis demonstrated that of the 17 patients with IVL, seven cases (41.18\%) exhibited c-Myc cleavage (Fig. 2D), eight cases (47.06\%) exhibited
Bcl-2 cleavage (Fig. 2E), seven cases (41.18\%) exhibited Bcl-6 cleavage (Fig. 2F) and 16 cases $(94.12 \%)$ exhibited positive IgH gene rearrangement (Fig. 3). 
ONCOLOGY LETTERS 20: 43, 2020

5

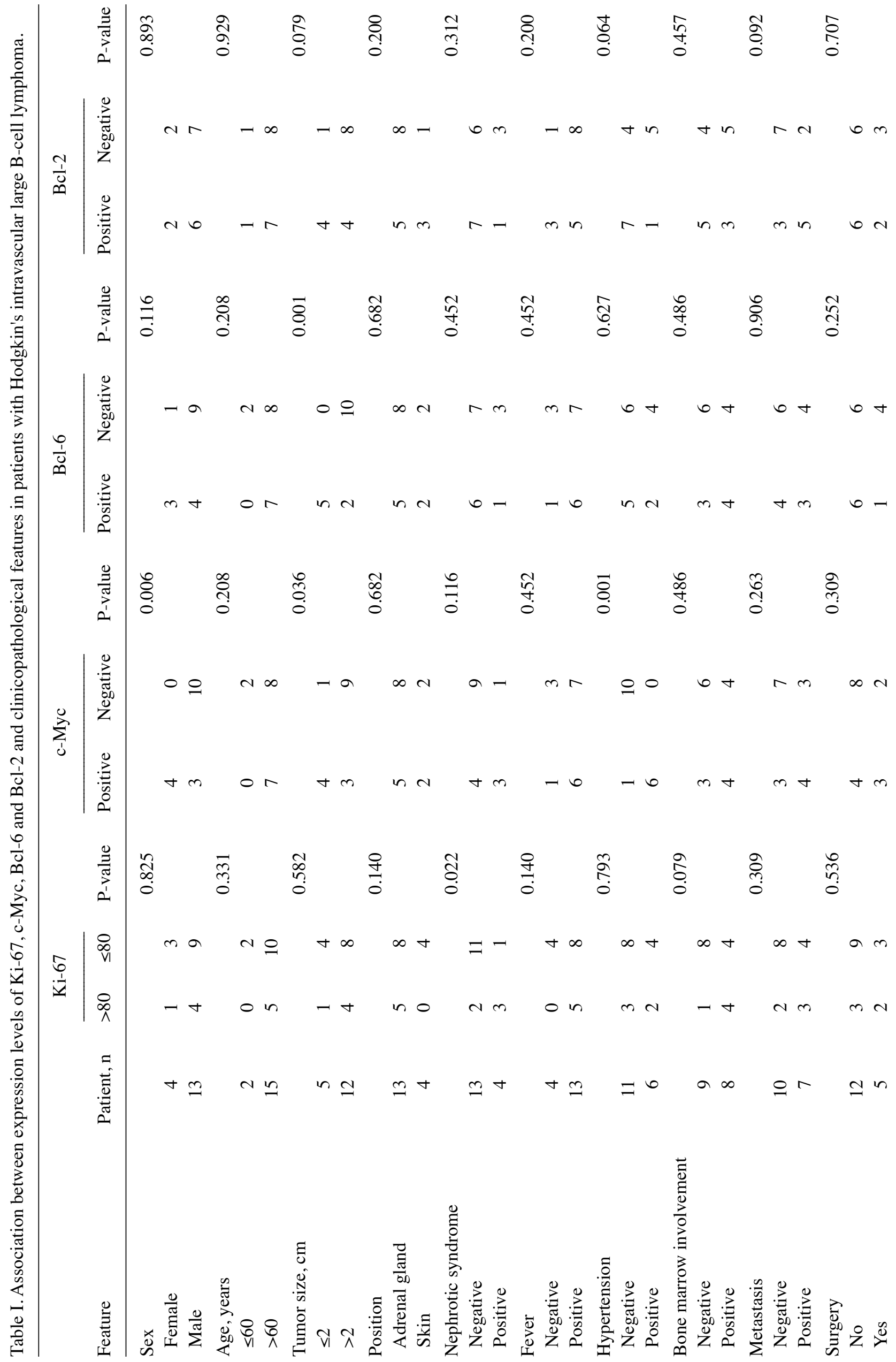




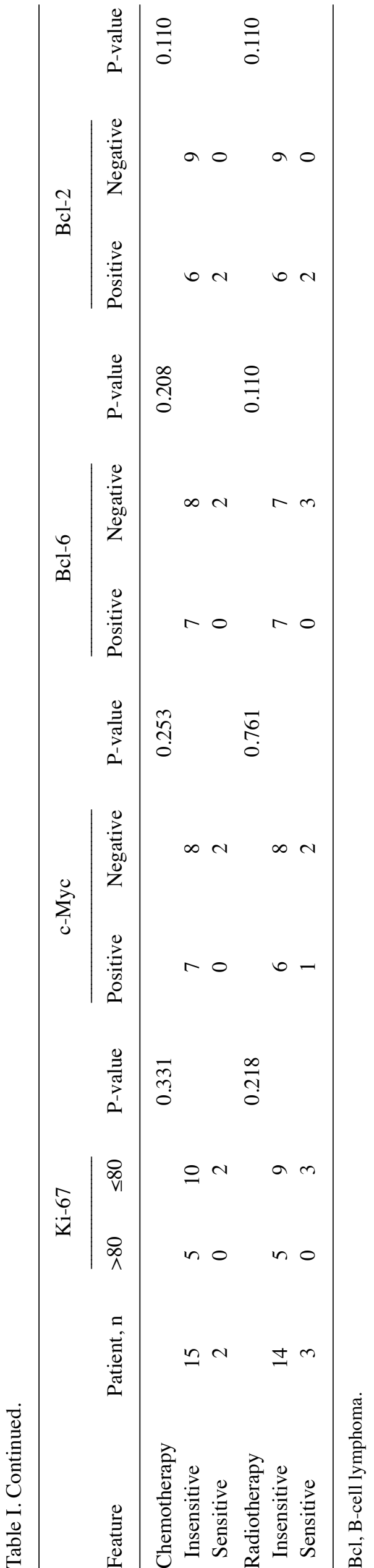

Association between Ki-67, c-Myc, Bcl-2 and Bcl-6 expression and clinicopathological features. The association between expression levels of c-Myc, Bcl-2 and Bcl-6 and clinicopathological features are presented in Table I. FISH analysis demonstrated that c-Myc exhibited statistical significance with regards to sex, hypertension status and tumor size (all $\mathrm{P}<0.05$ ), while the difference in Bcl-6 expression among tumor groups with different tumor sizes was statistically significant $(\mathrm{P}<0.05)$.

Association between clinicopathological features and prognosis. The follow-up period was 7.5-24.8 months, from the date of diagnosis of the disease (January 2019). The median follow-up time was 13.6 months. A total of 12 patients (70.6\%) died during the follow-up period. The average survival time was 13.2 months, and the overall survival rates of the 3 years were 75.6, 20.6 and $0.00 \%$, respectively. The 1- and 2-year survival rates in the low $\mathrm{Ki}-67$ expression group $(\leq 80 \%)$ were 83.3 and $31.3 \%$, respectively, which were significantly higher than those (60 and 0\%) in the high Ki-67 expression group $(>80 \%)(\mathrm{P}<0.05$; Fig. 4A). The survival time of the positive c-Myc group (the 1- and 2-year survival rates were 66.7 and $0.7 \%$, respectively) was significantly shorter compared with the negative group (the 1- and year 2-year survival rates were 81.80 and $35.1 \%$, respectively) $(\mathrm{P}<0.05$; Fig. 4B). The survival time of the positive Bcl-2 group (the 1- and 2-year survival rates were 53.70 and $0.93 \%$, respectively) was significantly shorter compared with the negative group (the 1- and year 2-year survival rates were 65.80 and $25.10 \%$, respectively). Furthermore, the survival time of the positive Bcl-6 group (the 1- and 2-year survival rates were 68.70 and $0.80 \%$, respectively) was significantly shorter compared with the negative group (the 1- and year 2-year survival rates were 80.80 and $33.10 \%$, respectively) $(\mathrm{P}<0.05$; Fig. 4C and D). The results demonstrated no significant association between survival time and age, sex, tumor size, tumor location, nephrotic syndrome, fever, hypertension, metastasis, bone marrow invasion, radiotherapy, chemotherapy and $\operatorname{IgH}$ rearrangement (all $\mathrm{P}>0.05$; Fig. 5A-L).

Clinicopathological features. The clinicopathological features were included as variables in the Cox regression model analysis, which demonstrated that the prognosis of the patients was associated with the Ki-67 proliferation index, c-Myc cleavage and Bcl-6 cleavage. Multivariate regression analysis demonstrated that the Ki-67 proliferation index was an independent risk factor for prognosis (survival time), as presented in Table II.

\section{Discussion}

IVL is a rare subtype of diffuse large B-cell lymphoma, characterized by selective growth of lymphoma cells in the small vascular cavity. It was first reported by Pfleger and Tappeiner in 1959 as hemangiomatosis proliferation syndrome, and is thought to have originated from the endothelium $(1,2)$. In 1982, Ansell et al detected immunoglobulins on the surface of tumor cells, suggesting that this was the origin of lymphocytes. In 1985, leukocyte common antigen was detected on the surface of tumor cells, and in 1986, Wick et al confirmed its lymphoma-like characteristics. Other historical appellations include hemangioendothelioma proliferation syndrome, 

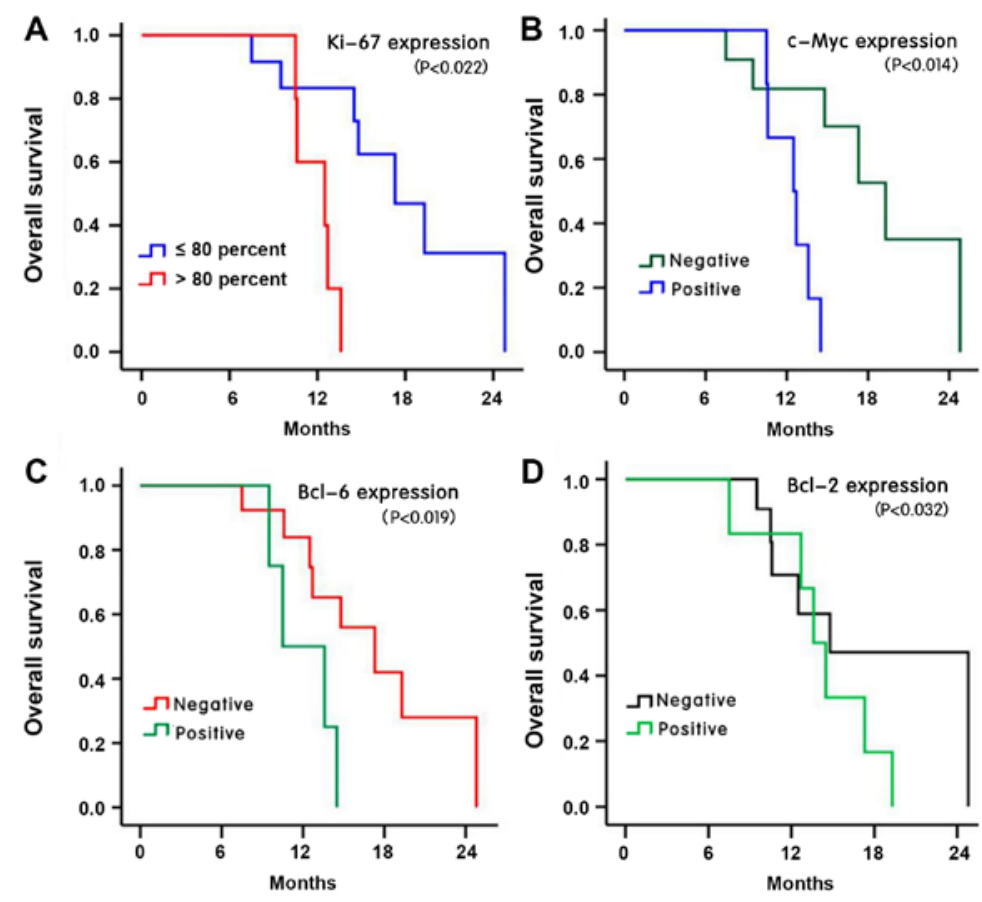

Figure 4. Kaplan-Meier survival curves for (A) Ki-67, (B) c-Myc, (C) Bcl-6 and (D) Bcl-2 of patients with Hodgkin's intravascular large B-cell lymphoma.
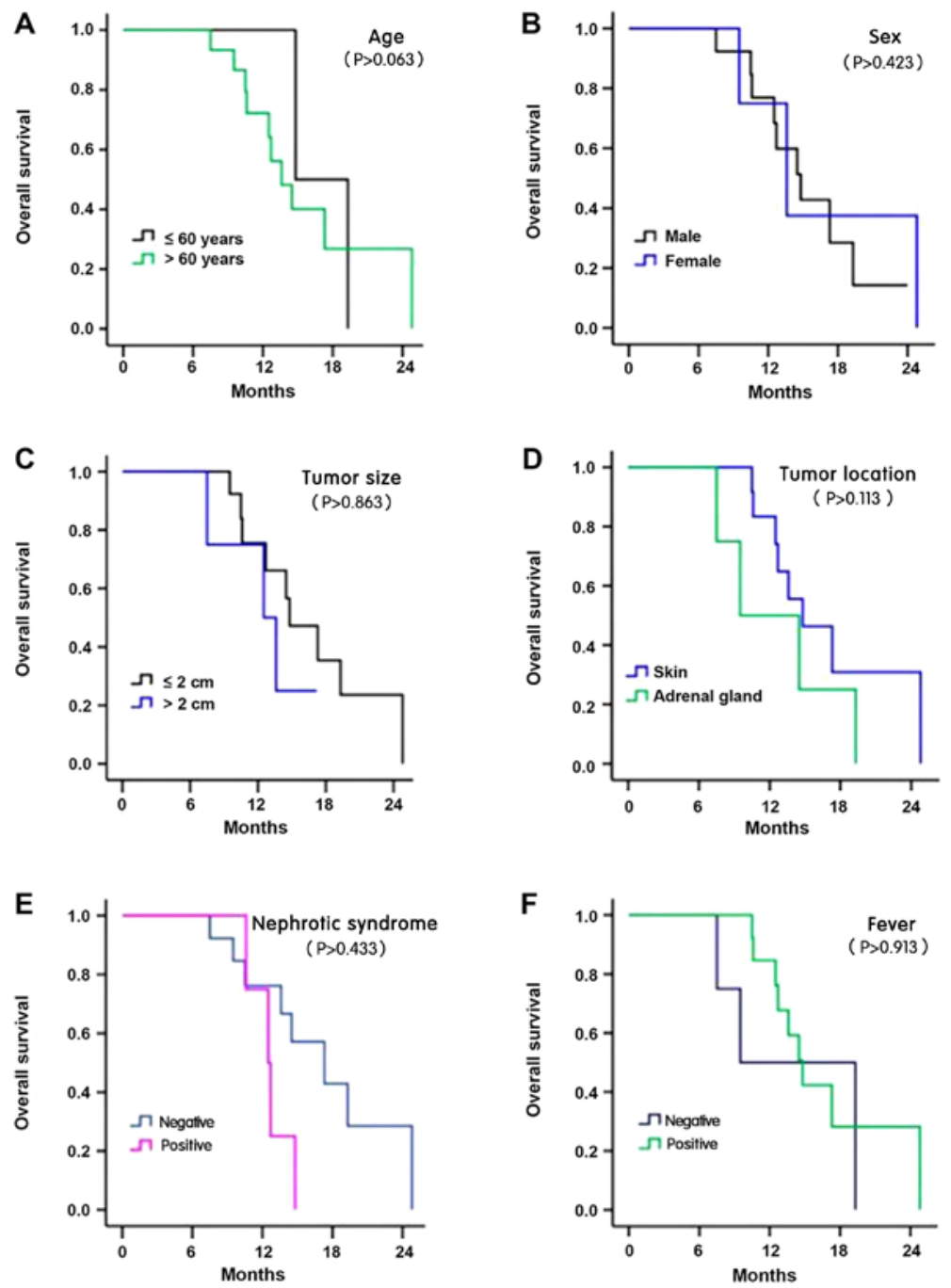

Figure 5. Survival analysis of patients with Hodgkin's intravascular large B-cell lymphoma. The Kaplan-Meier curves of (A) different age, (B) sex, (C) tumor size, (D) tumor location, and (E) nephrotic syndrome, and with or without (F) fever. 

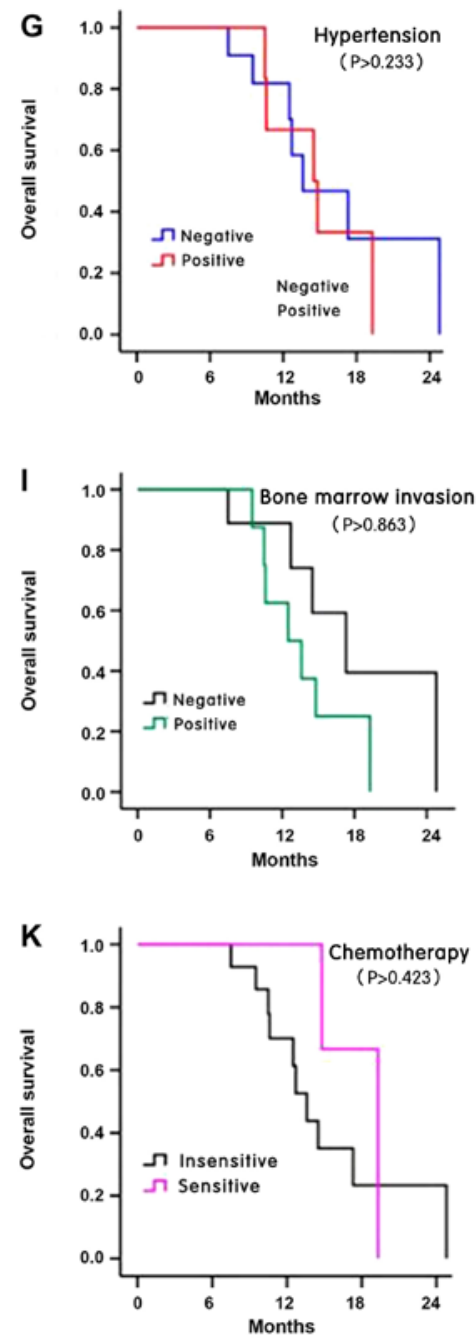
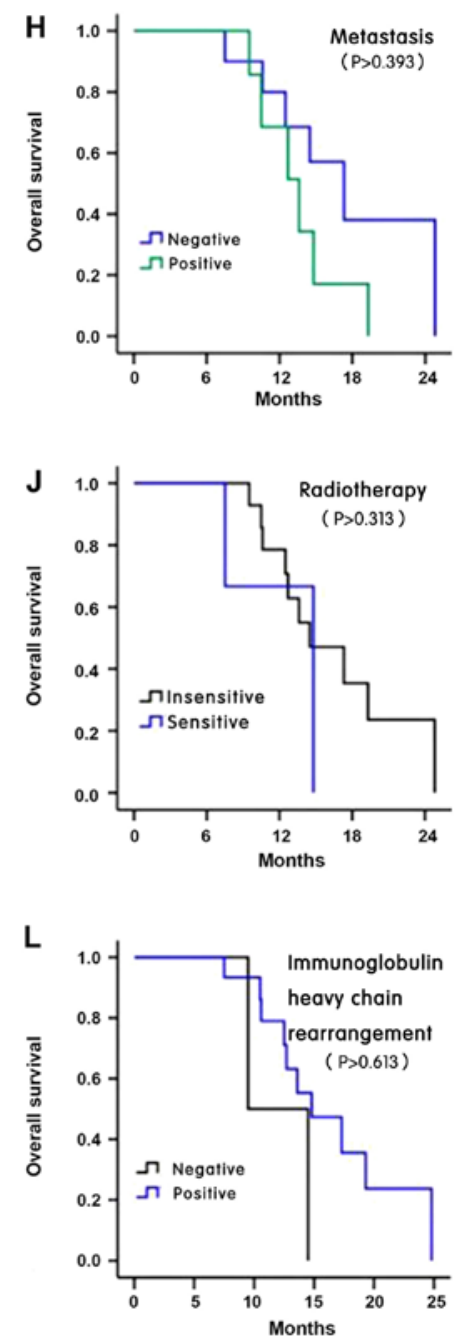

Figure 5. Continued. The Kaplan-Meier curves with or without (G) hypertension, (H) metastasis, (I) bone marrow invasion, (J) radiotherapy, (K) chemotherapyand, and (L) immunoglobulin heavy chain rearrangement.

malignant hemangioendothelioma, malignant endothelial proliferation, intravascular lymphoma, angiophilic endothelial (intravascular) lymphoma (Kiel's classification), angiophilic large cell lymphoma (Luke-Collins's classification) and diffuse large B-cell lymphoma (2-4). According to the recent WHO classification (2), IVL is a rare subtype of extranodal diffuse large B-cell lymphoma and has independent disease entity (2-8). Due to the fact that proliferating tumor cells only invade the small vessels and blood capillaries of different organs, they demonstrate a diversity and non-specificity of clinical symptoms, and the cause of disease still remains unclear (7-9). As there is no lymph node enlargement, no detection of abnormal peripheral blood and no notable abnormality in the bone marrow in the early stages of disease, early diagnosis was difficult, and the majority of cases were diagnosed in the late stages of disease or during autopsy (1). Fluorodeoxyglucose $\left({ }^{18} \mathrm{~F}-\mathrm{FDG}\right) \mathrm{PET} / \mathrm{CT}$ ha been reported to be beneficial for early diagnosis (9). As the incidence of the disease has increased in previous years, researchers in Japan have proposed the concept of 'Asian variants', based on the pathological features of large intravascular B-cell lymphoma (8). It has previously been reported that that the majority of tumors occur in middle-aged men, primarily aged $40-80$ years, $80 \%$ of which are $>60$ years of age (8). Among the 17 patients with IVL in the present study, 13 were men and four were women, aged 38-82 years (median age, 59 years; mean age, 57.2 years), indicating that the male patients had a younger age of onset, which was consistent with previous findings (7-9). A previous study demonstrated that the majority of patients in Europe and the United States possess tumors involved in the central nervous system and skin, which primarily invade the kidney, lung, adrenal gland, skin and soft tissue, and rarely involve the lymph nodes. Asian patients were dominated by hemophilic syndrome and bone marrow invasion (10). Of the patients in the present study, 13 cases were observed in the adrenal gland, while four cases were reported on the skin, and eight cases included bone marrow invasion, which was consistent with previous findings $(2,8)$. The clinical symptoms of each patient were not uniform, obvious or typical, and the majority of symptoms were observed following physical examination. The final diagnoses were confirmed following histopathological analysis. A previous study demonstrated that there are often symptoms of fever of unknown origin, weight loss or systemic inflammation of the whole body for a long period of time. Disease progression results in more central system injuries, including nephrotic syndrome, hypertension, leukopenia and pancytopenia, increased levels of serum lactic 
Table II. Univariate and Multivariate Cox regression analysis on the prognosis of patients with Hodgkin's intravascular large B-cell lymphoma.

\begin{tabular}{|c|c|c|c|c|}
\hline \multirow[b]{2}{*}{ Feature } & \multicolumn{2}{|c|}{ Univariate analysis } & \multicolumn{2}{|c|}{ Multivariate analysis } \\
\hline & $\mathrm{HR}(95 \% \mathrm{CI})$ & P-value & $\mathrm{HR}(95 \% \mathrm{CI})$ & P-value \\
\hline Ki-67 proliferation ( $>80$ vs. $\leq 80$ ) & $7.610(1.413-40.989)$ & 0.018 & $7.610(1.413-40.989)$ & 0.018 \\
\hline c-Myc expression (Positive vs. Negative) & $7.146(1.373-37.184)$ & 0.019 & $20.783(3.782-27.451)$ & 1.657 \\
\hline Bcl-6 expression (Positive vs. Negative) & $4.138(1.022-16.747)$ & 0.046 & $29.126(14.300-46.900)$ & 0.845 \\
\hline
\end{tabular}

HR, hazard ratio; CI, confidence interval; Bcl, B-cell lymphoma.

dehydrogenase and interleukin 6 (IL-6), recurrence and even death. Furthermore, the diversity of clinical symptoms (alongside atypical symptoms) can lead to delayed diagnosis $(10,11)$. In the present study, there were 13 cases of fever, six cases of hypertension, four cases of nephrotic syndrome, nine cases of increased serum lactic dehydrogenase, seven cases of increased IL-6 expression, eight cases of bone marrow invasion and seven cases of distant metastasis. Fever, hypertension, nephrotic syndrome, increased serum lactic dehydrogenase, increased IL-6 expression, bone marrow invasion and distant metastasis were analyzed via univariate, multivariate and survival analysis, and were demonstrated to not be statistically significant. A larger sample size is required for future studies.

In the present study Fisher's exact test was used to determine the difference between c-Myc, $\mathrm{Bcl}-2$ and $\mathrm{Bcl}-6$ expression and clinicopathological features. FISH analysis revealed that the presence of c-Myc, regardless of sex, hypertension status or different tumor size, Bcl-6 was also significantly different between the groups, with regards to tumor size. Multivariate analysis demonstrated that the Ki-67 proliferation index was an independent risk factor for prognosis (survival time). The positive rate of $\mathrm{Ki}-67$ was $>60 \%$, among which the rates in 11 cases were $>70 \%$, and the rates in five cases were $>80 \%$. High Ki-67 expression had certain clinical significance for the diagnosis and prognosis of IVL. The Ki67 proliferation index of different types of tumor has a certain guiding significance for the prognosis of patients, including IVL (10-12). The 1- and 2-year survival rates of the low Ki-67 expression group were 83.30 and $31.30 \%$, respectively, which was significantly higher compared with the high expression group (60.00 and $0.00 \%$, respectively) To the best of our knowledge, the effect of Ki-67 expression on survival rate has not yet been investigated, thus further verification is required by increasing the sample size. It has been reported that when one, two or three of the FISH-c-Myc, Bcl-2 or Bcl-6 proteins break at the same time, it suggests poor prognosis (11-13), which is inconsistent with the results of the present study. This may be due to the small sample size used in the present study. Previous studies have reported that $\mathrm{IgH}$ heavy chain gene clonal rearrangement was is observed in IVL tumor cells $(12,13)$, and chromosome translocation has also been exhibited in a few cases (13). Deficiency of the adhesion molecules, CD29 (B1 integrin) and CD54 (ICAM) in IVL tumor cells may be associated with their inability to break free from blood vessels. These adhesion molecules, which are involved in the migration of intravascular leukocytes, have been considered a factor in intravascular localization and can assist in diagnosis (14-17). In the present study, the rearrangement rate of large B-cell lymphoma was $>85 \%$, while 16 cases in this group were rearranged, which was consistent with the currently published literature. However, the molecular mechanism of IVL generation may be multifaceted and requires further investigation.

The present study was not without limitations. Firstly, the sample size used was too small. However, as IVL is an extremely rare disease it was difficult to attain sufficient samples. Regardless, a total of 17 cases of IVL were successfully collected, analyzed and summarized over a 10-year period, aiming to improve the understanding of the disease, and to provide a basis for clinical diagnosis and treatment.

IVL has a unique histopathological manifestation, and is primarily located in small vessels of organs or tissues (including arterioles, venules and capillaries, some of which are in medium vessels) filled with a large number of heteromorphic lymphocytic-like cells. The majority of tumor cells are round, large in volume, or irregular slightly nuclei; the chromatin is often aggregated, and small nucleolar and mitotic images can be observed (16-18). In the present study, the tissue originated from the adrenal gland and the skin, and the vasculature was observed to be CD34(+), CD20(+) and PAX-5(+).

IVL is characterized by the selective proliferation of tumor cells in the lumen of blood vessels (particularly capillaries), the majority of which are derived from NK/T cells. NK/T cell lymphomas account for only 10-15\% of IVLs. Morphologically, vascular dilatation, a large lumen, distribution of mononuclear cells with large nuclei and 1-2 small nucleoli may be observed. Many heteromorphic lymphocytic-like cells are present (which are large with a rich cytoplasm, with round, oval or irregular nuclei with dense chromatin) and thrombi may be present in the vascular cavity (16-19). Immunophenotypic CD3(+), CD56(+), GrB(+) and EBER(+) in situ hybrids have also been observed (14). However, in the present study, immunophenotypic CD3, CD56, GrB and EBER expression in situ was negative.

Intravascular lymphoid retention is a benign lymphoid lesion with chronic inflammatory changes and fibrosis that may lead to local lymphatic compression and lymphoid retention. The morphology of the tissue was similar to that of IVL, demonstrating a single $\mathrm{T}$ cell that was immunophenotypical; however, the heteromorphism of the cells was not notable, exhibiting isolated lesions and no marked malignant 
clinical manifestations. Immunophenotyping confirmed that the vessels were dilated lymphoid vessels $(2,12,14,17,18)$. The lymphocytes were primarily $\mathrm{T}$ cells, and the Ki-67-positive index was generally $<10 \%(15-19)$. Ki-67 expression in all patients of the present study was $>60 \%$, of which 11 cases were $>70 \%$. In anaplastic large cell lymphoma or other types of DLBCL, primary lesions in the blood infiltrate the surrounding tissues. However, intravascular lesions are only part of the tumor, while IVL cells only selectively proliferate in the blood vessels $(11,19)$. The immunophenotypes of the two can be distinguished by the distribution of tumor cells $(20,21)$. The clinical manifestations of extramedullary leukemia are similar to those of IVL, and include fever, hepatosplenomegaly and multiple organ failure; however, the peripheral hemogram of leukemia is abnormal, whereas the peripheral blood image of IVL is not $(16-18,20)$. A variety of myeloid antigens are expressed $(6,18,22)$; however, no tumor-cell expression of CD33, CD34 or CD117 was noted in the present study. Intravascular metastases, such as metastatic tumors and metastatic melanoma, are common primary lesions in vascular metastasis. Immunolabelling CKpan, HMB-45 and CD20 is useful for differential diagnosis (23-26); however, the expression of CKpan, HMB-45 and CD20 was not immunohistochemically identified in the present study.

In conclusion, the results of the present study demonstrated that IVL acts invasively and that the course of the disease progresses rapidly. The prognosis of the majority of patients was poor; the mean survival time was 6-9 months, whereas the survival time following non-active treatment was only 3-5 months, and the response to chemotherapy and radiotherapy was poor. Only two of the patients were sensitive to chemotherapy, while three cases were sensitive to radiotherapy. Previous studies have demonstrated that a mesna/ifosfamide, mitoxantrone and etoposide regimen is an effective treatment for recurrent and refractory non-Hodgkin's lymphoma $(13,14,19,27)$. It has some advantages in improving survival outcome and the adverse reactions of the digestive tract $(26,27)$; however, whether the regime is also effective against IVL has not yet been fully investigated.

\section{Acknowledgements}

Not applicable.

\section{Funding}

No funding was received.

\section{Availability of data and materials}

The datasets used and/or analyzed during the present study are available from the corresponding author upon reasonable request.

\section{Authors' contributions}

YL, YM and HZ were major contributors of data collection, data analysis and manuscript writing. XZ and JS were responsible for manuscript preparation, study design, data analysis and article finalization. All authors read and approved the final manuscript.

\section{Ethics approval and consent to participate}

The present study was approved by the Ethics Committee of Yantai Yuhuangding Hospital (Yantai, China; approval no. 2019-42681) and written informed consent was provided by all participants.

\section{Patient consent for publication}

Not applicable.

\section{Competing interests}

All authors declare that they have no competing interests.

\section{References}

1. Khojeini EV and Song JY: Intravascular large B-cell lymphoma presenting as interstitial lung disease. Case Rep Pathol 2014: 928065, 2014.

2. Sabattini E, Bacci F, Sagramoso C and Pileri SA: WHO classification of tumours of haematopoietic and lymphoid tissues in 2008: an overview. Pathologica 102: 83-87, 2010

3. Ferreri AJ, Dognini GP, Campo E, Willemze R, Seymour JF Bairey O, Martelli M,De Renz AO,DoglioniC, Montalbán C, et al: Variations in clinical presentation, frequency of hemophagocytosis and clinical behavior of intravascular lymphoma diagnosed in different geographical regions. Haematologica 92: 486-492, 2007.

4. Hope CB and Pincus LB: Primary cutaneous B-cell lymphomas with large cell predominance - primary cutaneous follicle center lymphoma, diffuse large B-cell lymphoma, leg type and intravascular large B-cell lymphoma. Semin Diagn Pathol 34: 85-98, 2017.

5. Lazzari I, Galetti C, Corvalli G, Bernardi R, Gianotti G, Sagramoso C and Calogero P: Intravascular large B-cell lymphoma as a cause of terminal acute respiratory distress syndrome: atypical presentation of a rare disease. Aging Clin Exp Res 30: 97-99, 2018.

6. Murase T, Yamaguchi M, Suzuki R, Okamoto M, Sato Y, Tamaru J, Kojima M, Miura I, Mori N, Yoshino T and Nakamura S: Intravascular large B-cell lymphoma (IVLBCL): A clinicopathologic study of 96 cases with special reference to the immnophenotypic heterogeneity of CD5. Blood 109: 478-485, 2007.

7. Shimada K, Kosugi H, Narimatsu H, Shimada S, Suzuki T, Ito M, Kinoshita T, Mori N and Naoe T: Sustainde remission after rituximab-containing chemotherapy for intravascular large B-cell lymphoma. J Clin Exp Hematol 48: 25-28, 2008.

8. Chen Y, Ding C, Lin Q, Yang K, Li Y and Chen S: Primary intravascular large B-cell lymphoma of the lung: A review and case report. J Thorace Dis 6: E242-E245, 2014.

9. Liu CL, Lai N, Zhou Y, Li S, Chen R and Zhang N: Intravascular large B-cell lymphoma confirmed by lung biopsy. Int J Clin Exp Pathol 7: 6301-6306, 2014.

10. Kohan AA, Paganini L, Biedak P, Arma JI, Dalurzo MC and Garcia-Monaco RD: Pulmonary intravascular lymphoma detected by FDG PET-CT: A case report. Rev Esp Med Nucl Imagen Mol 32: 318-320, 2013.

11. Bhargava P, Siddiqui F, Aggarwal B, Moore BE and Elble RJ: A unique case of intravascular lymphoma mimicking encephalomyeloradiculo neuropathy. Neurologist 20: 18-21, 2015.

12. Colavolpe C, Ebbo M, Trousse D, Khibri H, Franques J, Chetaille B, Coso D, Ouvrier MJ, Gastaud L, Guedj E and Schleinitz N: FDG-PET/CT is a pivotal imaging modality to diagnose rare intravascular large B-cell lymphoma: Case report and review of literature. Hematol Oncol 33: 99-109, 2015.

13. Nixon BK, Kussick SJ, Carlon MJ and Rubin BP: Intravascular large B-cell lymphoma involving hemangiomas: An unusual presentation of a rare neoplasm. Mod Pathol 18: 1121-1126, 2005. 
14. Cerroni L, Massone C, Kutzner H, Mentzel T, Umbert P and Kerl H: Intravascular large T-cell or NK-cell lymphoma: A rare variant of intravascular large cell lymphoma with frequent cytotoxic phenotype and association with Epstein-Barr virus infection. Am J Surg Pathol 32: 891-898, 2008.

15. Ardighieri L, Lonardi S, Vermi W, Medicina D, Cerroni L and Facchetti F: Intralymphatic atypical T-cell proliferation in a cutaneous hemangioma. J Cutan Pathol 37: 497-503, 2010.

16. Baum CL, Stone MS and Liu V: Atypical intravascular CD30+ $\mathrm{T}$ cell proliferation following trauma in a healthy 17 -year-old male: First reported case of a potential diagnostic pitfall and literature review. J Cutan Pathol 36: 350-354, 2009.

17. Xie JL, Shi Y, Zhou XG, Jin Y, Zheng XD and Wei XJ: Intralymphatic accumulation of lymphocytes mimicking intravascular lymphomatosis. Zhonghua Bing Li Xue Za Zhi 39: 518-521, 2010 (In Chinese).

18. Katayama K, Tomoda K, Ohya T, Asada H, Ohbayashi $\mathrm{C}$ and Kimura H: Ground-glass opacities and a solitary nodule on chest in intravascular large B-cell lymphoma. Respirol Case Rep 3: 108-111, 2015.

19. Yu H, Chen G, Zhang RX and Jin X: Primary intravascular large B-cell lymphoma of lung: A report of one case and review. Diagn Pathol 7: 70, 2012.

20. Maekawa T, Kornime M, Murata S, Fukushima N and Ohtsuki M: Random skin biopsy of patients with intravascular large B-cell lymphoma associated with thrombocytopenia and coagulation abnormalities: Proposal of a modified biopsy method. J Dermatol 42: 318-321, 2015.
21. Mahasneh T, Harrington Z, Williamson J, Alkhawaja D, Duflou J and Shin JS: Intravascular large B-cell lymphoma complicated by invasive pulmonary aspergillosis: A rare presentation. Respirol Case Rep 2: 67-69, 2014.

22. Willemze R, Jaffe ES, Burg G, Cerroni L, Berti E, Swerdlow SH Ralfkiaer E, Chimenti S, Diaz-Perez JL, Duncan LM, et al: WHO-EORTC classification for cutaneous lymphomas. Blood 105: 3768-3785, 2005.

23. Wenxue W: Progress in diagnosis and treatment of intravascular lymphomas. Modern Oncol 14: 1624-1627, 2006.

24. Chen M, Qiu B, Kong J and Chen J: Angiotropic T cell lymphoma. Chin Med J (Engl) 111: 762-764, 1998 .

25. Kobayashi H, Abe Y, Miura D, Narita K, Kitadate A, Takeuchi M and Matsue K: Limited efficacy of high-dose methotrexate in patients with neurolymphomatosis. Int J Hematol 109: 286-291, 2019.

26. Donald JS, Barnthouse N and Chen DL: Rare variant of intravascular Large B-cell lymphoma with hemophagocytic syndrome. Clin Nucl Med 43: e125-e126, 2018.

27. Ogasawara T, Ebata N, Hamasaki J, Marshall S, Kawauchi K, Ohshima K, Mori N and Sakura H: BCL2, BCL6, and MYC-positive intravascular large B-cell lymphoma presenting with bilateral adrenal gland lesions. Rinsho Ketsueki 60: 570-576, 2019 (In Japanese). 